\title{
Einstein equations for an asymmetric brane-world
}

\author{
Richard A. Battye ${ }^{1}$, Brandon Carter $^{2}$, Andrew Mennim ${ }^{1}$ and Jean-Philippe Uzan $^{3}$ \\ ${ }^{1}$ Department of Applied Mathematics and Theoretical Physics, Centre for Mathematical Sciences \\ University of Cambridge, Wilberforce Road, Cambridge, CB3 0WA, UK \\ ${ }^{2}$ Départment d'Astrophysique Relativiste et de Cosmologie, UMR 8629 du CNRS, \\ Observatoire de Paris, 92195 Meudon Cedex, France \\ ${ }^{3}$ Laboratoire de Physique Théorique, UMR 8627 du CNRS, \\ Université de Paris XI, Bâtiment 210, 91405 Orsay Cedex, France
}

(10 May 2001)

\begin{abstract}
We consider a brane-world of co-dimension one without the reflection symmetry that is commonly imposed between the two sides of the brane. Using the coordinate-free formalism of the GaussCodacci equations, we derive the effective Einstein equations by relating the local curvature to the matter on the brane in the case when its bare tension is much larger than the localized matter, and hence show that Einstein gravity is a natural consequence of such models in the weak field limit. We find agreement with the recently derived cosmological case, which can be solved exactly, and point out that such models can be realized naturally in the case where there is a minimally coupled form field in the bulk.
\end{abstract}

\section{INTRODUCTION}

Much recent work has focused on the idea that our 4 dimensional universe might be a 3-brane in a higher dimensional spacetime [1.22]. Although the initial motivation was to use the size of the extra dimensions to set the gravitational and electroweak scales equal in the higher dimensional space, hence "solving" the hierarchy problem, possibly the most striking result is that, under certain circumstances, higher dimensional gravity can appear from the point of view of an observed localized on the brane to be similar to ordinary Einstein gravity, with (i) the Newtonian inverse square law in the non-relativistic regime [3] above a given length scale and (ii) a cosmology compatible with the standard picture [4,5] but with an extra term contributed by the Weyl tensor of the bulk. The conditions on the bulk spacetime geometry required to recover the Einstein equations on the brane were studied in ref. [6].

The simplest relevant model [3] consists of a single hypermenbrane (i.e. brane of codimension one) with positive bare tension located in a bulk spacetime that is asymptotically anti-de-Sitter (adS) with reflection symmetry imposed along the extra dimension by hand. Matter localized to the brane is assumed to be a distributional source localised by a $\delta$-function. It was shown in ref. [3] that the gravitational fluctuations in this model, which has an (effectively infinite) extra dimension, are those of a spin-two graviton. This model is not without its problems, for instance, the apparent incompatibility with supersymmetry [7] (see, however, [8] for supersymmetrisations of [2]) and instability to unknown effects from the bulk 9, 10], but it serves as an interesting starting point for any investigation of the viability of scenarios with extra dimensions. Probing the assumptions of this model is the basis of our discussion here.

Most of the works have focused on a 3-brane embedded in a 5 dimensional spacetime with a reflection symmetry along the extra dimension [3 5, 9]. This was originally motivated by the Hořava-Witten theory [11] in which 11 dimensional M-theory on an orbifold that is the product of a smooth manifold and $S^{1} / Z_{2}$ - a circle with points identified by reflection symmetry about a diameter. This can be reduced to a 5 dimensional effective theory with 3-branes at the orbifold fixed points. These 3-branes inherit gauge groups from string theory which can produce the standard model gauge group on one of the branes. However, it should be emphasized that $D$-branes do not have to be supported at an orbifold fixed point [12].

Although originally physically motivated, these two assumptions (of dimensionality and reflection symmetry) owe their popularity to their convenience. To derive the Einstein equations on the brane, one needs to evaluate the extrinsic curvature of the brane in terms of the matter content of the theory, which in the case of a 5 dimensional $Z_{2}$-symmetric spacetime, is completely determined by the junction conditions. The relevant Darmois-Israel junction conditions relate the discontinuity of the extrinsic curvature to the matter content of the brane (see, for example, [9]), allowing the 4 dimensional geometry of the brane to be calculated using the Gauss-Codacci relations. In more general cases where one has more transverse dimensions the Gauss-Codacci equation relating the Ricci tensor of the brane to the geometry of the bulk can be generalized (see ref. [13]) but there is no straightforward generalisation of the Darmois-Israel junction conditions that apply to the hypersurface supported case. On the other hand, when the $Z_{2}$-symmetry hypothesis is relaxed, the Darmois-Israel junction conditions alone are not sufficient to determine the extrinsic curvature, so one needs to complement them with a dynamical equation for the brane motion [13, 14]. Despite these technical problems, some models with more than one extra dimensions have been studied [15] as well as 
some specific models in which the $Z_{2}$-symmetry was broken in an ad hoc way [16] by gluing two adS spacetimes with different cosmological constants. Recently, a very natural mechanism for breaking the $Z_{2}$-symmetry by means of a gauge form field has been proposed [14] and the Friedmann equations derived in this particular case [17]. We also note that some models with "moving branes" [18] have been considered, which share some properties with non-reflection symmetric brane model.

In this article, we relax the assumption of reflection symmetry and investigate the gravitational effects experienced by observers on the brane. To do this we choose the approach of constructing the effective Einstein equation from the Gauss-Codacci equations, as done in ref. [9] for the standard Randall-Sundrum model, and extended to the more general case where there is a scalar field in the bulk in refs 19,20. This should be compared and contrasted with the original [3] methods, which involve constructing an equation in the linearized approximation for the so-called graviton wave function, and higher energy modes due the effects of the extra dimension (see also ref. [21]); such methods should be equally applicable in the asymmetric case, but we do not consider them here.

As we noted above, the added complication in the case of an asymmetric model is that more general junction conditions 14 must be applied to the extrinsic curvature at the brane, which take into account the motion of the brane through the bulk spacetime. We first describe these conditions for a co-dimension one brane-world in section III. Using the Gauss equation, we shall show in section III that when the bare tension of the brane is large compared to that the matter localized on the brane, the results of ref. [9] can be generalized, with only modifications to the effective cosmological and gravitational constant terms, and that in principle higher order corrections can also be computed. This analysis shows that the effective gravitational excitations in the weak field limit are those of a massless spin-two graviton, as in the reflection symmetric case, but with more complicated higher order contributions from the bulk Weyl tensor. In section [V] we show that the Codacci equation governs possible transfer of energy momentum between the brane and the bulk. In the usual cases, it implies that the brane supported part of the energy momentum is conserved by itself.

Most work has focused on either the weak gravity as we do here, or the cosmological case of a homogeneous and isotropic brane-world in which one effectively probes gravity on large scales [5.,A. Even when the $Z_{2}$ symmetry is broken, the dynamic equation for the cosmological case can be solved exactly [17]. In section \$, we show that the effective gravitational and cosmological constants obtained in the perturbative analysis carried out here agree with those obtained in the preceeding homogeneous cosmological analysis [17].

In section VI, we consider the possibility that the absence of reflection symmetry might result from the coupling of the brane to an (antisymmetric) gauge form field that has bulk support. Outside the distributional matter source on the brane, an $(n-1)$-form in an $n$ dimensional spacetime has no dynamics: its effect is just to modify the cosmological constant in the bulk on either side, with a discontinuity across the brane.

Whereas the earlier sections treat the general case in an $n$ dimensional bulk, finally, in section VII we consider the specific case of a 3-brane coupled to a 4 -form gauge field in a 5 dimensional bulk. We shall comment on the implications of our work for linearized gravity and cosmological expansion.

\section{HYPERMEMBRANE DYNAMICS}

We shall consider the case of an $n-1$ dimensional brane in a $n$ dimensional spacetime (referred to as the bulk). As standard matter (and thus observers) are confined to the brane in such models, the relevant geometry for comparison with observations will be that of the brane world-volume rather than the one of the bulk. This will be given in terms of internal coordinates, $\sigma^{a}$, by embedding functions $x^{\mu}=\bar{x}^{\mu}\left(\sigma^{a}\right)$. The induced brane world-volume metric, $\gamma_{a b}$, with respect to the internal coordinates can be expressed in terms of the bulk metric and embedding functions by

$$
\gamma_{a b}=g_{\mu \nu} \frac{\partial \bar{x}^{\mu}}{\partial \sigma^{a}} \frac{\partial \bar{x}^{\nu}}{\partial \sigma^{b}}
$$

where greek and latin indices run respectively from 0 to $n-1$ and from 0 to $n-2$. The contravariant inverse of $\gamma_{a b}$ and the associated internal Ricci tensor, $R^{a b}$, can be mapped into bulk tensors, given with respect to bulk coordinates, by

$$
\eta^{\mu \nu}=\gamma^{a b} \frac{\partial \bar{x}^{\mu}}{\partial \sigma^{a}} \frac{\partial \bar{x}^{\nu}}{\partial \sigma^{b}} \quad \text { and } \quad \bar{R}^{\mu \nu}=R^{a b} \frac{\partial \bar{x}^{\mu}}{\partial \sigma^{a}} \frac{\partial \bar{x}^{\nu}}{\partial \sigma^{b}}
$$

the first of which is known as the first fundamental tensor of the world-volume. The corresponding mixed version $\eta^{\mu}{ }_{\nu}$ acts as a projection operator, projecting bulk tensors at a point on the brane into tensors tangential to the brane and defines a complementary orthogonal projection operator given by $\perp^{\mu}{ }_{\nu} \equiv \delta^{\mu}{ }_{\nu}-\eta^{\mu}{ }_{\nu}$. The above definitions are valid for a brane world-volume of arbitrary co-dimension but, in the case where the co-dimension is one, this orthogonal tensor can be expressed in terms of the unit normal, $n^{\mu}$ to the brane by $\perp^{\mu}{ }_{\nu}=n^{\mu} n_{\nu}$. 
The internal Ricci tensor can be related to the Ricci tensor of the bulk, $\mathcal{R}_{\mu \nu}$, by the Gauss equation 13 (see also ref. [22]) as

$$
\bar{R}_{\mu \nu}=\frac{n-3}{n-2} \mathcal{R}_{\rho \sigma} \eta_{\mu}^{\rho} \eta_{\nu}^{\sigma}+\frac{1}{n-2} \mathcal{R}_{\rho \sigma} \eta^{\rho \sigma} \eta_{\mu \nu}-\frac{1}{n-1} \mathcal{R} \eta_{\mu \nu}+K K_{\mu \nu}-K_{\mu}{ }^{\rho} K_{\nu \rho}+\overline{\mathcal{W}}_{\mu \nu}
$$

where $\overline{\mathcal{W}}_{\mu \nu}$ is obtained by a surface projection from the Weyl tensor of the bulk spacetime, $\mathcal{C}_{\mu \rho \nu \sigma}$ :

$$
\overline{\mathcal{W}}_{\mu \nu} \equiv-\mathcal{C}_{\mu \rho \nu \sigma} \perp^{\rho \sigma}
$$

Since it is the trace free part of the bulk Riemann tensor, one can show that

$$
\overline{\mathcal{W}}_{\mu}^{\mu}=0, \quad \overline{\mathcal{W}}_{\mu \nu} \perp^{\mu \nu}=0 .
$$

The extrinsic curvature $K_{\mu \nu}$ is defined as

$$
K_{\mu \nu} \equiv-\eta_{\nu}^{\sigma} \eta_{\mu}^{\alpha} \nabla_{\alpha} n_{\sigma}
$$

where $\nabla_{\mu}$ is the covariant derivative associated with $g_{\mu \nu}$. Assuming that $n_{\sigma}$ is smoothly continued into the bulk along geodesics (i.e., that $n^{\alpha} \nabla_{\alpha} n_{\mu}=0$ ), it reduces to

$$
K_{\mu \nu}=-\nabla_{\mu} n_{\nu}
$$

(using the conventions of ref. [23]). In a Gaussian normal coordinate system where the coordinate $\zeta$ measures the orthogonal distance from the brane (implying that $n_{\mu}=\nabla_{\mu} \zeta$ ), the background metric takes the standard form

$$
\mathrm{d} s_{[n]}^{2} \equiv g_{\mu \nu} \mathrm{d} x^{\mu} \mathrm{d} x^{\nu}=\mathrm{d} \zeta^{2}+\mathrm{d} \bar{s}_{[n-1]}^{2},
$$

with

$$
\mathrm{d} \bar{s}_{[n-1]}^{2} \equiv \eta_{\mu \nu} \mathrm{d} x^{\mu} \mathrm{d} x^{\nu}=\gamma_{a b} \mathrm{~d} \sigma^{a} \mathrm{~d} \sigma^{b}
$$

and the extrinsic curvature is

$$
2 K_{\mu \nu} \mathrm{d} x^{\mu} \mathrm{d} x^{\nu}=-\frac{\mathrm{d}}{\mathrm{d} \zeta} \gamma_{a b} \mathrm{~d} \sigma^{a} \mathrm{~d} \sigma^{b}
$$

Although the bulk metric $g_{\mu \nu}$ is everywhere continuous, its derivative along $n^{\mu}$ may not be continuous across the brane. This implies that $\mathcal{R}_{\mu \nu}, K_{\mu \nu}$ and $\overline{\mathcal{W}}_{\mu \nu}$ may also be discontinuous. We use square brackets to denote the jump of a particular quantity across the brane, defined with respect to the orientation of the normal $n_{\mu}$, and angled brackets to denote its mean. As an example, we decompose the extrinsic curvature tensor $K_{\mu \nu}$ as

$$
\left[K_{\mu \nu}\right] \equiv K_{\mu \nu}^{+}-K_{\mu \nu}^{-} \quad \text { and } \quad\left\langle K_{\mu \nu}\right\rangle \equiv \frac{1}{2}\left(K_{\mu \nu}^{+}+K_{\mu \nu}^{-}\right)
$$

where the + superscript denotes the side of the brane into which the normal, $n_{\mu}$, points (the $\zeta>0$ region). Since $\eta_{\mu \nu}$ must be smooth, $\bar{R}_{\mu \nu}$ must be continuous across the brane so that the jump and average of the Gauss equation (3) are given by

$$
\begin{aligned}
\left\langle\bar{R}_{\mu \nu}\right\rangle & =\bar{R}_{\mu \nu}=\left\langle\mathcal{F}_{\mu \nu}\right\rangle+\left\langle K K_{\mu \nu}\right\rangle-\left\langle K_{\mu}{ }^{\rho} K_{\nu \rho}\right\rangle, \\
{\left[\bar{R}_{\mu \nu}\right] } & =0=\left[\mathcal{F}_{\mu \nu}\right]+\left[K K_{\mu \nu}\right]-\left[K_{\mu}{ }^{\rho} K_{\nu \rho}\right]
\end{aligned}
$$

where we have separated the contributions arising from the extrinsic curvature and the ones from the bulk geometry, which are encapsulated in $\mathcal{F}_{\mu \nu}$ defined as

$$
\mathcal{F}_{\mu \nu} \equiv \frac{n-3}{n-2} \mathcal{R}_{\rho \sigma} \eta_{\mu}^{\rho} \eta_{\nu}^{\sigma}+\frac{1}{n-2} \mathcal{R}_{\rho \sigma} \eta^{\rho \sigma} \eta_{\mu \nu}-\frac{1}{n-1} \mathcal{R} \eta_{\mu \nu}+\overline{\mathcal{W}}_{\mu \nu}
$$

One can easily check that \langle\rangle and [ ] satisfy the two product relations

$$
\langle A B\rangle=\langle A\rangle\langle B\rangle+\frac{1}{4}[A][B] \quad \text { and } \quad[A B]=\langle A\rangle[B]+[A]\langle B\rangle,
$$


and, hence, to deduce $\bar{R}_{\mu \nu}$ we need to compute $\left[K_{\mu \nu}\right]$ and $\left\langle K_{\mu \nu}\right\rangle$ in terms of the stress energy of the brane, $\bar{T}_{\mu \nu}$.

The extrinsic curvature of the brane is related to the stress energy $\bar{T}^{\mu \nu}$ (with $\bar{T}^{\mu \nu} n_{\mu}=0$ ) supported on the brane and to the force $\left(\bar{f}^{\mu} \equiv \bar{f} n^{\mu}\right)$ exerted on the brane by the bulk stress energy tensor by the generalisation of Newton's second law 14

$$
\bar{T}^{\mu \nu}\left\langle K_{\mu \nu}\right\rangle=\bar{f},
$$

relating the contraction of the mean extrinsic curvature and $\bar{T}^{\mu \nu}$ (reducing to mass times acceleration for a point particle) to the force, $\bar{f}$, exerted by the bulk. This force is given in terms of the bulk energy-momentum tensor, $T_{\mathrm{ba}}^{\mu \nu}$, by

$$
\bar{f}=-\left[T_{\mathrm{ba}}^{\mu \nu}\right] n_{\mu} n_{\nu},
$$

which can be related to the bulk Ricci tensor $\mathcal{R}_{\mu \nu}$ by the $n$ dimensional Einstein equations. We should note that the dynamical equation (16) can be derived from (13), but that it has more general applicability, for instance when gravity is not present, since it is just a consequence of energy-momentum conservation. For our purposes, we find that it is more economical to use (13). In addition, the brane energy-momentum, $\bar{T}^{\mu \nu}$, determines the jump in the extrinsic curvature across the brane via the well-known junction conditions

$$
\left[K \eta_{\mu \nu}-K_{\mu \nu}\right]=\kappa_{[n]} \bar{T}_{\mu \nu},
$$

where $\kappa_{[n]}$ is the gravitational constant of the $n$ dimensional spacetime related to the $n$ dimensional gravitational constant $G_{[n]}$ by

$$
\kappa_{[n]} \equiv(n-2) \Omega^{[n-2]} G_{[n]},
$$

and $\Omega^{[q]}$ is the area of the $q$-sphere (for instance $\Omega^{[2]}=4 \pi, \Omega^{[3]}=2 \pi^{2} \ldots$ ).

In brane-world models, it is common to interpret the stress energy of the brane as the sum of a bare tension and observed matter, the former being the dominant contribution (except, perhaps, in the very early universe) so that we shall decompose it as

$$
\bar{T}_{\mu \nu}=-\overline{\mathcal{T}}_{\infty} \eta_{\mu \nu}+\overline{\mathcal{T}}_{\mu \nu}=\overline{\mathcal{T}}_{\infty}\left(\varepsilon_{\mu \nu}-\eta_{\mu \nu}\right)
$$

where $\overline{\mathcal{T}}_{\infty}(\neq 0)$ is the bare tension of the brane, and $\varepsilon_{\mu \nu} \equiv \overline{\mathcal{T}}_{\mu \nu} / \overline{\mathcal{T}}_{\infty}$ is the dimensionless, energy-momentum localized to the brane. In section III we will assume that $\left\|\varepsilon_{\mu \nu}\right\| \ll 1$ to make a series expansion. The jump condition (18) inverts to give us

$$
[K]=\kappa_{[n]} \overline{\mathcal{T}}_{\infty}\left(1+\frac{1-\varepsilon}{n-2}\right) \quad \text { and } \quad\left[K_{\mu \nu}\right]=\kappa_{[n]} \overline{\mathcal{T}}_{\infty}\left(\varepsilon_{\mu \nu}+\frac{1-\varepsilon}{n-2} \eta_{\mu \nu}\right)
$$

where $\varepsilon=\varepsilon^{\mu}{ }_{\mu}$ and the brane dynamical equation (16) becomes

$$
\overline{\mathcal{T}}_{\infty}\left(\varepsilon^{\mu \nu}-\eta^{\mu \nu}\right)\left\langle K_{\mu \nu}\right\rangle=\bar{f} \quad \text { with } \quad 2 \kappa_{[n]} \bar{f}=\left[2 \mathcal{R}^{\mu \nu} \eta_{\mu \nu}-\mathcal{R}\right]=[\mathcal{F}] .
$$

Here, we have made use of the bulk Einstein equations and the definition (14) of $\mathcal{F}_{\mu \nu}$ to express the force, $\bar{f}$, in terms of $[\mathcal{F}]$. Indeed, this equation is a scalar equation and alone cannot completely determine $\left\langle K_{\mu \nu}\right\rangle$ therefore, as stressed above, we also have to use equation (13) which can be rewritten as

$$
\left[\mathcal{F}_{\mu \nu}\right]=\left\langle K_{\mu}{ }^{\rho}\right\rangle\left[K_{\rho \nu}\right]+\left[K_{\mu}{ }^{\rho}\right]\left\langle K_{\rho \nu}\right\rangle-\langle K\rangle\left[K_{\mu \nu}\right]-[K]\left\langle K_{\mu \nu}\right\rangle
$$

The general brane Einstein equation is then given by

$$
\begin{aligned}
\bar{R}_{\mu \nu}= & \frac{n-3}{n-2}\left\langle\mathcal{R}_{\rho \sigma}\right\rangle \eta^{\rho}{ }_{\mu} \eta^{\sigma}{ }_{\nu}+\frac{1}{n-2}\left\langle\mathcal{R}_{\rho \sigma}\right\rangle \eta^{\rho \sigma} \eta_{\mu \nu}-\frac{1}{n-1}\langle\mathcal{R}\rangle \eta_{\mu \nu}+\left\langle\overline{\mathcal{W}}_{\mu \nu}\right\rangle+\frac{1}{4}\left([K]\left[K_{\mu \nu}\right]-\left[K_{\mu}{ }^{\rho}\right]\left[K_{\nu \rho}\right]\right) \\
& +\langle K\rangle\left\langle K_{\mu \nu}\right\rangle-\left\langle K_{\mu}{ }^{\rho}\right\rangle\left\langle K_{\nu \rho}\right\rangle .
\end{aligned}
$$

\footnotetext{
${ }^{1}$ These are almost universally referred to as the Israel junction conditions [24], but this extrinsic curvature method was used much earlier by the French mathematician George Darmois 25. Even earlier work on junctions in spacetimes was done by Lanczos [26] and Sen 27]. See 24] for further references.
} 
The equations we have stated allow us, in principle, to get expression for the $n-1$ dimensional Ricci scalar in terms of the matter in the spacetime, that is, both the matter supported throughout the bulk and that supported on the brane. In the most general case, equation (13) is not easy to invert. In subsequent sections we solve this problem in two ways: firstly, in the case of arbitrary matter content, by a series expansion in $\varepsilon_{\mu \nu}$ and, secondly, in the case of cosmological models where the brane matter is a perfect fluid and for which an exact solution can be found.

Before trying to disentangle this set of equations, let us point out that imposing the reflection symmetry implies that $\left[K_{\mu \nu}\right]=2 K_{\mu \nu}$ and $\left\langle K_{\mu \nu}\right\rangle=0$ which makes equation (22) and (23) trivial and allows to directly express $\left[K_{\mu \nu}\right]$ in terms of the brane stress-energy tensor $\bar{T}_{\mu \nu}$ by only making use of the Israel junction conditions (18). The general brane equation (24) then reduces to the one derived in ref. [9].

As a first interpretation of equation (24), let us note that $\vec{R}_{\mu \nu}$ has three origins: (i) an "induced" part coming from the geometry of the bulk, depending on the bulk Ricci and Weyl tensors, (ii) a part coming from the jump in the extrinsic curvature across the brane, and (iii) a part coming from the average of the extrinsic curvature at the brane. In the reflection symmetric models, only (i) and (ii) contribute but, in the most general case, there is a combination of all these effects. In a companion paper [28], we shall investigate the other extreme limiting case were only (i) and (iii) have an effect.

\section{SERIES SOLUTION FOR GENERAL MATTER CONTENT}

We are now in a position to obtain a series solution for $K_{\mu \nu}$ in terms of $\varepsilon_{\mu \nu}$ using (13) by means of successive approximations, or equivalently expanding to the relevant order and separating by order. The product relations (15) and the Israel conditions (21) allow us to rewrite (13) as

$$
\frac{1}{\kappa_{[n]} \overline{\mathcal{T}}_{\infty}}\left[\mathcal{F}_{\mu \nu}\right]=2 \varepsilon_{(\mu}{ }^{\rho}\left\langle K_{\nu) \rho}\right\rangle-\left(1-\frac{1-\varepsilon}{n-2}\right)\left\langle K_{\mu \nu}\right\rangle-\langle K\rangle\left(\varepsilon_{\mu \nu}+\frac{1-\varepsilon}{n-2} \eta_{\mu \nu}\right) .
$$

So, if we expand $\left\langle K_{\mu \nu}\right\rangle$ as

$$
\left\langle K_{\mu \nu}\right\rangle=\left\langle K_{\mu \nu}^{(0)}\right\rangle+\left\langle K_{\mu \nu}^{(1)}\right\rangle+\mathcal{O}\left(\varepsilon^{2}\right)
$$

where $K_{\mu \nu}^{(p)}$ is of order $\mathcal{O}\left(\varepsilon^{p}\right)$ in $\varepsilon_{\mu \nu}$, and then substitute into (25) then the zeroth order term gives

$$
\left\langle K_{\mu \nu}^{(0)}\right\rangle=-\frac{1}{\kappa_{[n]} \overline{\mathcal{T}}_{\infty}(n-3)}\left((n-2)\left[\mathcal{F}_{\mu \nu}\right]-\frac{1}{2}[\mathcal{F}] \eta_{\mu \nu}\right) .
$$

Substituting this back into (25), the first-order term gives

$$
\begin{gathered}
\left\langle K_{\mu \nu}^{(1)}\right\rangle=\frac{1}{\kappa_{[n]} \overline{\mathcal{T}}_{\infty}(n-3)^{2}}( \\
(n-2) \varepsilon^{\rho \sigma}\left[\mathcal{F}_{\rho \sigma}\right] \eta_{\mu \nu}+(n-2) \varepsilon\left[\mathcal{F}_{\mu \nu}\right]-2(n-2)^{2} \varepsilon_{(\mu}{ }^{\rho}\left[\mathcal{F}_{\nu) \rho}\right] \\
\left.+\frac{1}{2}(n-1)(n-2)[\mathcal{F}] \varepsilon_{\mu \nu}-\frac{1}{2}(n-1) \varepsilon[\mathcal{F}] \eta_{\mu \nu}\right) .
\end{gathered}
$$

This process could, of course, be iterated to calculate higher order terms of the series expansion (26). Motivated by the fact that $\overline{\mathcal{W}}_{\mu \nu}$ is traceless, we decompose the bulk effects, $\mathcal{F}_{\mu \nu}$, into the trace and a trace-free part, $\omega_{\mu \nu}\left(\omega^{\mu}{ }_{\mu}=0\right)$, so that

$$
\left[\mathcal{F}_{\mu \nu}\right]=\frac{2 \kappa_{[n]} \bar{f}}{n-1} \eta_{\mu \nu}+\kappa_{[n]}\left[\omega_{\mu \nu}\right]
$$

from which, using expression (22) one can deduce that

$$
\kappa_{[n]}\left[\omega_{\mu \nu}\right]=\frac{n-3}{n-2}\left[\mathcal{R}_{\rho \sigma}\right]\left(\eta_{\mu}^{\rho} \eta_{\nu}^{\sigma}-\frac{1}{n-1} \eta^{\rho \sigma} \eta_{\mu \nu}\right)+\left[\overline{\mathcal{W}}_{\mu \nu}\right] .
$$

Equations (27) and (28) then simplify to 


$$
\begin{aligned}
& \overline{\mathcal{T}}_{\infty}\left\langle K_{\mu \nu}^{(0)}\right\rangle=-\frac{\bar{f}}{(n-1)} \eta_{\mu \nu}-\frac{n-2}{n-3}\left[\omega_{\mu \nu}\right], \\
& \overline{\mathcal{T}}_{\infty}\left\langle K_{\mu \nu}^{(1)}\right\rangle=\frac{\bar{f}}{(n-1)}\left((n-2) \varepsilon_{\mu \nu}-\varepsilon \eta_{\mu \nu}\right)+\frac{n-2}{(n-3)^{2}}\left(\varepsilon^{\rho \sigma}\left[\omega_{\rho \sigma}\right] \eta_{\mu \nu}+\varepsilon\left[\omega_{\mu \nu}\right]-2(n-2) \varepsilon_{(\mu}{ }^{\rho}\left[\omega_{\nu) \rho}\right]\right) .
\end{aligned}
$$

With this series expansion for $\left\langle K_{\mu \nu}\right\rangle$, we can evaluate the Ricci tensor of the brane (24) by substituting (21) and (31) to eliminate the $K$ terms in favour of $\varepsilon_{\mu \nu}, \bar{f}$ and $\left[\omega_{\mu \nu}\right]$

$$
\begin{aligned}
\bar{R}_{\mu \nu}= & \left(\frac{1}{n-1}\langle\mathcal{F}\rangle+\frac{\kappa_{[n]}^{2} \overline{\mathcal{T}}_{\infty}^{2}}{4(n-2)}+\frac{(n-2) \bar{f}^{2}}{(n-1)^{2} \overline{\mathcal{T}}_{\infty}^{2}}\right) \eta_{\mu \nu}+\left(\frac{\kappa_{[n]}^{2} \overline{\mathcal{T}}_{\infty}^{2}}{4(n-2)}-\frac{(n-2) \bar{f}^{2}}{(n-1)^{2} \overline{\mathcal{T}}_{\infty}^{2}}\right)\left((n-3) \varepsilon_{\mu \nu}-\varepsilon \eta_{\mu \nu}\right) \\
& +\kappa_{[n]}\left\langle\omega_{\mu \nu}\right\rangle+\frac{\bar{f}}{(n-1) \overline{\mathcal{T}}_{\infty}^{2}}\left[\omega_{\mu \nu}\right]-\frac{(n-2)^{2}}{(n-3)^{2} \overline{\mathcal{T}}_{\infty}^{2}}\left[\omega_{\mu}{ }^{\rho}\right]\left[\omega_{\rho \nu}\right]+\frac{2(n-2) \bar{f}}{(n-1)(n-3) \overline{\mathcal{T}}_{\infty}^{2}}\left(2(n-2) \varepsilon_{(\mu}{ }^{\rho}\left[\omega_{\nu) \rho}\right]-\varepsilon\left[\omega_{\mu \nu}\right]\right) \\
& +\frac{(n-2)^{2}}{(n-3)^{3} \overline{\mathcal{T}}_{\infty}^{2}}\left(2 \varepsilon\left[\omega_{\mu}{ }^{\rho}\right]\left[\omega_{\rho \nu}\right]-4(n-2) \varepsilon_{\rho}^{\sigma}\left[\omega_{(\mu}{ }^{\rho}\right]\left[\omega_{\nu) \sigma}\right]-4(n-2) \varepsilon_{(\mu}{ }^{\sigma}\left[\omega_{\nu)}{ }^{\rho}\right]\left[\omega_{\rho \sigma}\right]-(n-5) \varepsilon^{\rho \sigma}\left[\omega_{\rho \sigma}\right]\left[\omega_{\mu \nu}\right]\right) \\
& +\mathcal{O}\left(\varepsilon^{2}\right),
\end{aligned}
$$

and, taking the trace, we compute the Ricci scalar to be

$$
\begin{aligned}
\bar{R}= & \left(\langle\mathcal{F}\rangle+\frac{\kappa_{[n]}^{2} \overline{\mathcal{T}}_{\infty}^{2}(n-1)}{4(n-2)}+\frac{(n-2) \bar{f}^{2}}{(n-1) \overline{\mathcal{T}}_{\infty}^{2}}\right)+\left(2 \frac{(n-2) \bar{f}^{2}}{(n-1)^{2} \overline{\mathcal{T}}_{\infty}^{2}}-\frac{\kappa_{[n]}^{2} \overline{\mathcal{T}}_{\infty}^{2}}{2(n-2)}\right) \varepsilon-\frac{(n-2)^{2}}{(n-3)^{2} \overline{\mathcal{T}}_{\infty}^{2}}\left[\omega_{\rho \sigma}\right]\left[\omega^{\rho \sigma}\right] \\
& +\frac{4(n-2)^{2} \bar{f}}{(n-1)(n-3) \overline{\mathcal{T}}_{\infty}^{2}} \varepsilon_{\rho \sigma}\left[\omega^{\rho \sigma}\right]+\frac{2(n-2)^{2}}{(n-1)^{2} \overline{\mathcal{T}}_{\infty}^{2}}\left(\varepsilon\left[\omega_{\rho \sigma}\right]\left[\omega^{\rho \sigma}\right]-4(n-2) \varepsilon_{\nu}{ }^{\rho}\left[\omega^{\nu \sigma}\right]\left[\omega_{\rho \sigma}\right]\right)+\mathcal{O}\left(\varepsilon^{2}\right) .
\end{aligned}
$$

Now, we can deduce the Einstein tensor

$$
\bar{G}_{\mu \nu}=-\Lambda_{[n-1]} \eta_{\mu \nu}+\kappa_{[n-1]} \overline{\mathcal{T}}_{\mu \nu}+\mathcal{O}\left(\omega, \varepsilon^{2}\right),
$$

where the cosmological constant on the brane is given by

$$
\Lambda_{[n-1]}=\frac{n-3}{2}\left(\frac{\langle\mathcal{F}\rangle}{n-1}+\frac{\kappa_{[n]}^{2} \overline{\mathcal{T}}_{\infty}^{2}}{4(n-2)}+\frac{(n-2) \bar{f}^{2}}{(n-1)^{2} \overline{\mathcal{T}}_{\infty}^{2}}\right),
$$

and the gravitational constant, is identified to be

$$
\kappa_{[n-1]}=(n-3)\left(\frac{\kappa_{[n]}^{2} \overline{\mathcal{T}}_{\infty}}{4(n-2)}-\frac{(n-2) \bar{f}^{2}}{(n-1)^{2} \overline{\mathcal{T}}_{\infty}^{3}}\right) .
$$

It should be noted that these are exactly the same as found ref. [17] for the exact cosmological case with $n=5$, an issue to which we will return later.

This enables us to compare these brane-world theories with more traditional gravity theories, such as four dimensional general relativity or Brans-Dicke theory. From the brane Einstein equation (35) we can recover the results of ref. [9] in the physically interesting case of $n=5$ by setting $\bar{f}$ to zero. As with the reflection symmetric case, unequivocal interpretation of the result is problematic due to the contribution from bulk effects $\left\langle\omega_{\mu \nu}\right\rangle$, which is indeterminate from the point of view of observers on the brane. This is the case even in the reflection symmetric case [9: 10]; their interpretation being that these degrees of freedom correspond to the massive states of the higher dimensional graviton. In the more general case studied here, there is an additional indeterminacy arising from $\left[\omega_{\mu \nu}\right]$ terms, but we should note that this is no more than one would expect since one will have massive modes from both sides. In this respect, the asymmetric case is at least no worse than that with reflection symmetry.

\section{CODACCI EQUATION}

In addition to the Gauss equation the extrinsic curvature satisfies the Codacci equation, 


$$
\overline{\mathcal{D}}_{\nu} K-\overline{\mathcal{D}}_{\mu} K^{\mu}{ }_{\nu}=\mathcal{R}_{\rho \sigma} n^{\sigma} \eta^{\rho}{ }_{\nu}
$$

where $\overline{\mathcal{D}}_{\mu}$ denotes the covariant derivative associated to the induced brane metric $\eta_{\mu \nu}$. Using the jump conditions (21), this equation gives an equation for the divergence of the brane energy-momentum $\varepsilon_{\mu \nu}$

$$
\kappa_{[n]} \overline{\mathcal{T}}_{\infty} \overline{\mathcal{D}}_{\mu} \varepsilon^{\mu}{ }_{\nu}=-\left[\mathcal{R}_{\rho \sigma}\right] n^{\sigma} \eta^{\rho}{ }_{\nu} .
$$

This relates the four dimensional divergence of $\varepsilon_{\mu \nu}$ to the energy flow between the bulk and the brane, which is zero in the reflection symmetric case, but not necessarily so in general. Since energy is observed to be conserved to a high degree in the late universe this will place strong restrictions on the relevant components of the bulk Ricci tensor. However we should note that violating brane energy conservation in the early universe may indeed be desirable in order to alleviate cosmological puzzles such as the horizon and flatness problems. If the bulk spacetime is effectively dictated by two different bulk cosmological constants, as is the case when the asymmetry in the spacetime is generated by the form fields discussed in section V1, then the right hand side of (39) is zero and standard energy conservation on the brane is generic.

There is also an equation coming from the average of (38), which restricts the variation of $\left[\omega_{\mu \nu}\right]$, in a similar way to the Bianchi identities constrain $\left\langle\omega_{\mu \nu}\right\rangle$. Using our series solution from Section III, the zeroth order terms of this give us

$$
\frac{1}{\overline{\mathcal{T}}_{\infty}}\left(\frac{1}{2(n-1)} \overline{\mathcal{D}}_{\nu} \bar{f}+\frac{n-2}{n-3} \overline{\mathcal{D}}_{\mu}\left[\omega^{\mu}{ }_{\nu}\right]\right)=\left\langle\mathcal{R}_{\rho \sigma}\right\rangle n^{\sigma} \eta^{\rho}{ }_{\nu}
$$

Clearly, the first order corrections will be constrained by similar relations.

\section{EXACT SOLUTION FOR COSMOLOGICAL MODELS}

Of great interest are cosmological models which are spatially isotropic and homogeneous. This means that the brane matter content will have the specific isotropic and homogeneous form

$$
\overline{\mathcal{T}}_{\mu \nu}=\mathcal{U} u_{\mu} u_{\nu}-\mathcal{T}\left(u_{\mu} u_{\nu}+\eta_{\mu \nu}\right)=\overline{\mathcal{T}}_{\infty}\left[\hat{\rho} u_{\mu} u_{\nu}+\hat{p}\left(\eta_{\mu \nu}+u_{\mu} u_{\nu}\right)\right]
$$

where $\hat{\rho}$ and $\hat{p}$ are, respectively, the dimensionless ratios of the energy density and pressure to the bare brane tension, $\overline{\mathcal{T}}_{\infty}$, and $u_{\mu}$ is the unit timelike vector tangent to the brane (that is, $u^{\mu} u_{\mu}=-1$ and $\perp_{\mu \nu} u^{\nu}=0$ ) which is normal to the $(n-2)$-surfaces of isotropy and homogeneity (that is, to the spatial sections of our universe). In this case, equation (25) becomes

$$
\frac{1}{\kappa_{[n]} \overline{\mathcal{T}}_{\infty}}\left[\mathcal{F}_{\mu \nu}\right]=\left(\frac{\hat{\rho}+1}{n-2}+\hat{p}-1\right)\left\langle K_{\mu \nu}\right\rangle+2(\hat{\rho}+\hat{p})\left\langle K_{(\mu}{ }^{\rho}\right\rangle u_{\nu)} u_{\rho}-\left(\frac{\hat{\rho}+1}{n-2} \eta_{\mu \nu}+(\hat{\rho}+\hat{p}) u_{\mu} u_{\nu}\right)\langle K\rangle .
$$

This can be inverted exactly (see appendix $\mathrm{A}$ for details) to give

$$
\begin{aligned}
\kappa_{[n]} \overline{\mathcal{T}}_{\infty}\left(\frac{\hat{\rho}+1}{n-2}+\hat{p}-1\right)\left\langle K_{\mu \nu}\right\rangle= & {\left[\mathcal{F}_{\mu \nu}\right]+2 \frac{(\hat{\rho}+\hat{p})(n-2)}{(\hat{\rho}+1)(n-3)}\left[\mathcal{F}_{(\mu}{ }^{\rho}\right] u_{\nu)} u_{\rho}+\frac{(n-2)(\hat{\rho}+\hat{p})^{2}}{(n-3)(\hat{\rho}+1)^{2}}\left[\mathcal{F}_{\rho \sigma}\right] u^{\rho} u^{\sigma} u_{\mu} u_{\nu} } \\
& -\frac{(\hat{\rho}+\hat{p})(\hat{\rho}-(n-2) \hat{p}+n-1)}{2(\hat{\rho}+1)^{2}(n-3)}[\mathcal{F}] u_{\mu} u_{\nu} \\
& -\left(\frac{\hat{\rho}+\hat{p}}{(n-3)(\hat{\rho}+1)}\left[\mathcal{F}_{\rho \sigma}\right] u^{\rho} u^{\sigma}+\frac{(2 n-5) \hat{\rho}+(n-2) \hat{p}+n-3}{2(\hat{\rho}+1)(n-2)(n-3)}[\mathcal{F}]\right) \eta_{\mu \nu} .
\end{aligned}
$$

Making the split (29) of $\mathcal{F}_{\mu \nu}$ into its trace, $\bar{f}$, and its trace-free part, $\omega_{\mu \nu}$, we get

$$
\begin{aligned}
\overline{\mathcal{T}}_{\infty}\left\langle K_{\mu \nu}\right\rangle= & \frac{\bar{f}}{(n-1)(\hat{\rho}+1)^{2}}\left((\hat{\rho}+\hat{p})(n-2) u_{\mu} u_{\nu}-(\hat{\rho}+1) \eta_{\mu \nu}\right)+\left(\left[\omega_{\mu \nu}\right]+2 \frac{(\hat{\rho}+\hat{p})(n-2)}{(\hat{\rho}+1)(n-3)}\left[\omega_{(\mu}{ }^{\rho}\right] u_{\nu)} u_{\rho}\right. \\
& \left.+\frac{(n-2)(\hat{\rho}+\hat{p})^{2}}{(n-3)(\hat{\rho}+1)^{2}}\left[\omega_{\rho \sigma}\right] u^{\rho} u^{\sigma} u_{\mu} u_{\nu}-\frac{(\hat{\rho}+\hat{p})}{(n-3)(\hat{\rho}+1)}\left[\omega_{\rho \sigma}\right] u^{\rho} u^{\sigma} \eta_{\mu \nu}\right) \frac{n-2}{\hat{\rho}+(n-2) \hat{p}-n+3},
\end{aligned}
$$

and the trace of (44) has the relatively simple form 


$$
\overline{\mathcal{T}}_{\infty}\langle K\rangle=\frac{(n-2)(\hat{\rho}+\hat{p})-(n-1)(\hat{\rho}+1)}{(n-1)(\hat{\rho}+1)^{2}} \bar{f}-\frac{(\hat{\rho}+\hat{p})(n-2)}{(n-3)(\hat{\rho}+1)^{2}}\left[\omega_{\rho \sigma}\right] u^{\rho} u^{\sigma} .
$$

Equation (44) can be substituted into (24), along with the junction conditions, to give an exact expression for the $n-1$ dimensional Einstein tensor. As we have spatial isotropy and homogeneity, there is only one free Einstein equation, which it is convenient to take as the equation for the Ricci scalar, given by the trace of (24) as

$$
\bar{R}=\langle\mathcal{F}\rangle+\frac{1}{4}\left([K]^{2}-\left[K^{\mu \nu}\right]\left[K_{\mu \nu}\right]\right)+\langle K\rangle^{2}-\left\langle K^{\mu \nu}\right\rangle\left\langle K_{\mu \nu}\right\rangle .
$$

So the part of the Ricci scalar which does not depend on $\omega_{\mu \nu}$ is

$$
\begin{aligned}
\bar{R}= & \langle\mathcal{F}\rangle+\frac{\kappa_{[n]}^{2} \overline{\mathcal{T}}_{\infty}^{2}}{4(n-2)}(n-1+2(\hat{\rho}-(n-2) \hat{p})-(2(n-2) \hat{p}+(n-3) \hat{\rho}) \hat{\rho}) \\
& +\frac{(n-2) \bar{f}^{2}}{\overline{\mathcal{T}}_{\infty}^{2}(n-1)(\hat{\rho}+1)^{3}}((n-1)(\hat{\rho}+1)+2(n-2)(\hat{\rho}+\hat{p})) .
\end{aligned}
$$

It is simple to check for consistency with (34) by expanding the denominator of the last term keeping only the terms linear in $\hat{\rho}$ and $\hat{p}$. One finds that the relevant coefficients agree completely with the definitions of $\Lambda_{[n-1]}$ and $\kappa_{[n-1]}$ given by (36) and (37). Hence, we have confirmed the results of section III are correct to linearized order for an isotropic perfect fluid.

\section{SPECIFIC CASE OF A BULK FORM FIELD}

We now discuss a simple possible realization of our asymmetric brane-world scenario in terms of an $(n-1)$-form field $A^{\{n-1\}}{ }_{\nu_{1} \ldots \nu_{n-1}}$ which might naturally arise in some M-theory compactification which gives rise to the model. Such field couple to the brane world-volume via a generalized Wess-Zumino interaction and have field strength

$$
F_{\nu_{0} \nu_{1} \ldots \nu_{n-1}}^{\{n\}}=n \nabla_{\left[\nu_{0}\right.} A_{\left.\nu_{1} \ldots \nu_{n-1}\right]}^{\{n-1\}} .
$$

As discussed in ref. [14], this field strength can be written in terms of a single pseudo-scalar $F^{\{n\}}$ and the totally antisymmetric tensor $\epsilon_{\nu_{0} \ldots \nu_{n-1}}$. Moreover, the dynamics of this field are such that it will be constant except for jump at the brane which is proportional to their coupling, $e^{\{n\}}$, when the matter on the brane is $\delta$-function localized. Hence, effectively, one has a scenario with a discontinuous bulk cosmological constant such that

$$
\bar{f}=e^{\{n-1\}}\left\langle F^{\{n\}}\right\rangle=\kappa_{[n]}^{-1}\left[\Lambda_{[n]}\right] .
$$

If no other matter is present in the bulk, its Ricci tensor will then satisfy the following minimal coupling isotropy condition,

$$
\mathcal{R}_{\mu \nu}=\frac{1}{n} \mathcal{R} g_{\mu \nu},
$$

where $\mathcal{R}$ will necessarily be uniform on each side of the brane. This gives

$$
\mathcal{F}_{\mu \nu}=\frac{n-2}{n(n-1)} \mathcal{R} \eta_{\mu \nu}+\overline{\mathcal{W}}_{\mu \nu}
$$

Thus equation (29) implies that

$$
\omega_{\mu \nu}=\frac{\overline{\mathcal{W}}_{\mu \nu}}{\kappa_{[n]}} \quad \text { and } \quad \mathcal{F}=\frac{n-2}{n} \mathcal{R}
$$

Hence the right hand side of the four dimensional energy conservation equation (39) is zero so, if we consider the case where the bare tension, $\overline{\mathcal{T}}_{\infty}$, is constant, then we still have conservation of the energy-momentum tensor $\varepsilon_{\mu \nu}$.

We now consider cosmological solutions where the bulk has $(n-2)$ dimensional spatial surfaces of isotropy and homogeneity. With this minimally coupled form, it is possible to apply a generalized Birkhoff theorem to such a 
spacetime, as was demonstrated in ref. [29] and applied to this situation in ref. [17]. According to this theorem, the metric in the bulk will not just be homogeneous and isotropic but necessarily static, so that in suitable coordinates it will be expressible in the the form

$$
\mathrm{d} s_{[n]}^{2}=r^{2} \mathrm{~d} \ell^{2}+\frac{\mathrm{d} r^{2}}{\mathcal{V}}-\mathcal{V} \mathrm{d} t^{2},
$$

where $\mathrm{d} \ell^{2}$ is the (positive-definite) space metric of an $n-2$ dimensional sphere, plane or anti-sphere with constant curvature, $k$ say — which will be respectively positive, zero, or negative - and $\mathcal{V}$ is given on either side by an expression of the generalized Schwarzschild-de Sitter form

$$
\mathcal{V}=k-\frac{\mathcal{R}^{ \pm}}{n(n-1)} r^{2}-\frac{2 G_{[n]} \mathcal{M}^{ \pm}}{r^{n-3}}
$$

where $\mathcal{M}^{+}$and $\mathcal{M}^{-}$are constants, which will be interpretable, in the case of positive curvature with the standard normalization $k=1$, as representing the total asymptotic mass on either side.

As discussed in ref. [17], in this homogeneous and isotropic case, the line element can be re-written in terms of Gaussian normal coordinates as

$$
\mathrm{d} s_{[n]}^{2}=r^{2} \mathrm{~d} \ell^{2}+\mathrm{d} \zeta^{2}-\nu^{2} \mathrm{~d} \tau^{2},
$$

where the brane is located at $\zeta=0$ and the new time coordinate $\tau$ is constant on geodesics orthogonal to the brane. The function $\nu$ depends only on $\zeta$ and $\tau$, and can be normalized so that $\nu \rightarrow 1$ when $\zeta \rightarrow 0-$ that is, on the brane. This means that $\tau$ will simply be a measure of proper time within the brane. The corresponding limit $r(\tau, \zeta) \rightarrow a(\tau)$ defines a function, interpretable as a cosmological scale factor, which satisfies

$$
\left(\frac{\mathrm{d} a}{\mathrm{~d} \tau}\right)^{2}=\left(\frac{\partial \alpha}{\partial \zeta}\right)^{2}-\mathcal{V}
$$

In addition, $\nu$ must satisfy

$$
\nu \frac{\mathrm{d} a}{\mathrm{~d} \tau}=\frac{\partial r}{\partial \tau} .
$$

It follows that the first fundamental form, $\eta_{\mu \nu}$, is given in this case by

$$
\mathrm{d} \bar{s}_{[n-1]}^{2}=a^{2} \mathrm{~d} \ell^{2}-\mathrm{d} \tau^{2},
$$

and the second fundamental form, $K_{\mu \nu}$, is given by

$$
K_{\mu \nu} \mathrm{d} x^{\mu} \mathrm{d} x^{\nu}=-a \frac{\partial r}{\partial \zeta} \mathrm{d} \ell^{2}+\frac{\partial \nu}{\partial \zeta} \mathrm{d} \tau^{2} .
$$

Following the method described in ref. [23], it is possible, in the case of a spacetime with the line element given by (53) and (54), to derive an expression for the contribution of the bulk projected Weyl tensor, $\overline{\mathcal{W}}_{\mu \nu}$, which can be expressed as (see Appendix B for details)

$$
\overline{\mathcal{W}}_{\mu \nu} \mathrm{d} x^{\mu} \mathrm{d} x^{\nu}=\mathcal{W}^{ \pm}\left(a^{2} \mathrm{~d} \ell^{2}+(n-2) \mathrm{d} \tau^{2}\right)
$$

in which the Weyl scalars, $\mathcal{W}^{ \pm}$— which, of course, are not the trace of the Weyl tensor since this that would give zero - are related to the asymptotic mass on each side of the brane by

$$
\mathcal{W}^{ \pm}=(n-3) \frac{G_{[n]} \mathcal{M}^{ \pm}}{a^{n-1}} .
$$

Expression (61) leads to the usual interpretation of the Weyl contribution as a pseudo-radiation term when $n=5$. In terms of the preferred timelike unit vector, $u_{\mu}=-\nabla_{\mu} \tau$, used in the definition of (41), which specifies the cosmological rest frame, $\overline{\mathcal{W}}_{\mu \nu}$ can be written as

$$
\overline{\mathcal{W}}_{\mu \nu}=\mathcal{W}^{ \pm}\left(\eta_{\mu \nu}+(n-1) u_{\mu} u_{\nu}\right)
$$


and, therefore, substituting this into (44) gives us the expression for $\left\langle K_{\mu \nu}\right\rangle$,

$$
\begin{aligned}
\overline{\mathcal{T}}_{\infty}\left\langle K_{\mu \nu}\right\rangle= & \frac{\bar{f}}{(n-1)(\hat{\rho}+1)^{2}}\left((\hat{\rho}+\hat{p})(n-2) u_{\mu} u_{\nu}-(\hat{\rho}+1) \eta_{\mu \nu}\right) \\
& -\frac{[\mathcal{M}]}{\Omega^{[n-2]} a^{n-1}(\hat{\rho}+1)^{2}}\left((\hat{\rho}+(n-2) \hat{p}+n-1) u_{\mu} u_{\nu}+(\hat{\rho}+1) \eta_{\mu \nu}\right),
\end{aligned}
$$

which depends only on $\hat{\rho}, \hat{p}$ and on the two constant parameters $\bar{f}$ and $[\mathcal{M}]$. The trace of these equation has the simple form

$$
\overline{\mathcal{T}}_{\infty}\langle K\rangle=\frac{1-n-(2 n-3) \hat{\rho}-(n-2) \hat{p}}{(n-1)(\hat{\rho}+1)^{2}} \bar{f}-\frac{(n-2)(\hat{\rho}+\hat{p})}{\Omega^{[n-2]}(\hat{\rho}+1)^{2}}[\mathcal{M}] .
$$

These equations can then be substituted into (23) and (24) to derive the results found in ref. [17]. We have shown that, in the perfectly homogeneous case, $\left\langle\omega_{\mu \nu}\right\rangle \propto\langle\mathcal{M}\rangle$ and $\left[\omega_{\mu \nu}\right] \propto[\mathcal{M}]$, where $\mathcal{M}$ is the mass on any Schwarzschild black hole which resides in the bulk. In the original Randall-Sundrum scenario [3] the bulk spacetime is pure adS, so $\mathcal{M}$ would be zero.

\section{SUMMARY: 3-BRANE IN A FIVE DIMENSIONAL SPACETIME}

In this article, we have obtained the effective Einstein equations for a $n-1$ dimensional hypermembrane embedded in a $n$ dimensional spacetime without imposing any reflection symmetry along the extra dimension, hence generalizing the previous work of ref. [9]. We have found [see, for example, equation (24)] that the $n-1$ dimensional Ricci tensor has two origins, one coming from the bulk geometry and a second due to the breakdown of the reflection symmetry. In general, the closed set of equations is difficult to invert but we have shown that it is possible firstly, by means of a series expansion in the weak field limit in which the ordinary matter was a small contribution with respect to the brane tension and, secondly, exactly if the matter in the brane is a perfect fluid.

Now, to summarize our results let us consider the specific physically interesting case where $n=5$. We have shown is that in such a model the curvature tensor on the brane (35) can be written in the simple form

$$
\bar{G}_{\mu \nu}=-\Lambda_{[4]} \eta_{\mu \nu}+8 \pi G_{[4]} \overline{\mathcal{T}}_{\mu \nu},
$$

if one ignores $\mathcal{O}\left(\mathcal{T}^{2}\right)$ terms and the effects of the bulk modes, which we have argued will be related to higher dimensional massive modes in an exactly similar way to the case when the system is reflection symmetric. The relevant cosmological (36) and gravitational (37) constants are given, whatever the matter on the brane, by

$$
\Lambda_{[4]}=\frac{\langle\mathcal{F}\rangle}{4}+\frac{\kappa_{[5]}^{2} \overline{\mathcal{T}}_{\infty}^{2}}{12}+\frac{3 \bar{f}^{2}}{16 \overline{\mathcal{T}}_{\infty}^{2}},
$$

and

$$
\kappa_{[4]}=8 \pi G_{[4]}=\frac{\kappa_{[5]}^{2} \overline{\mathcal{T}}_{\infty}}{6}-\frac{3 \bar{f}^{2}}{8 \overline{\mathcal{T}}_{\infty}^{3}},
$$

which are exactly the same as derived in the cosmological case [17. Moreover, we have shown that the precise form of the cosmological expansion rate is the same once it is linearized to the relevant order. Finally, we have pointed out that, in such a scenario, an asymmetric brane-world is natural within a model where 4-form field is coupled to the brane via a generalized Wess-Zumino action. This puts on a more solid footing the earlier, more ad hoc work on this reflection symmetry breaking scenarios 16 .

Introducing the two mass scales $m_{\Lambda}^{ \pm}$, the inverse of which are length scales characterizing the bulk geometry on both sides,

$$
m_{\Lambda}^{ \pm} \equiv \pi G_{[5]} \overline{\mathcal{T}}_{\infty} \pm \frac{\bar{f}}{4 \overline{\mathcal{T}}_{\infty}}
$$

we can rewrite $(66-67)$ as 


$$
8 \pi G_{[4]}=\frac{6}{\overline{\mathcal{T}}_{\infty}} m_{\Lambda}^{+} m_{\Lambda}^{-} \quad \text { and } \quad 2 \Lambda_{[4]}=\frac{\langle\mathcal{F}\rangle}{2}+3\left\langle m_{\Lambda}^{2}\right\rangle .
$$

In the particular case of a maximally symmetric background, which is the case when a 4 -form field is coupled to the brane, $\mathcal{R}_{\mu \nu}=\mathcal{R} g_{\mu \nu} / n$ and we obtain that $\langle\mathcal{F}\rangle=(n-2)\langle\mathcal{R}\rangle / n$ so that for a five dimensional anti-de-Sitter spacetime $\langle\mathcal{F}\rangle=2\langle\Lambda\rangle$. The two mass scales $m_{\Lambda}^{ \pm}$need not only to be positive but large enough to lead to a viable cosmology [17].

The original result of Randall and Sundrum that five dimensional gravity can yields standard Einstein gravity on a brane with positive bare tension was a surprising one. It was mainly based on the fact that the extrinsic curvature was large enough to simulate the effect of a small compactification radius. Here, we have shown it generalizes to a brane which is not reflection symmetric provide the curvature radius on each side is small enough or, equivalently, that the mass scales, $m_{\Lambda}^{ \pm}$, are both large enough — who knows where it will end!

\section{ACKNOWLEDGEMENTS}

RAB is funded by PPARC and AM is funded by EPSRC. JPU would like to thank D. Steer and M. Ruiz-Altaba for discussions on moving branes while he was in Sils Maria, as well as E. Dudas and J. Mourad.

\section{APPENDIX A: CALCULATING $\left\langle K_{\mu \nu}\right\rangle$ FOR A PERFECT FLUID}

To invert equation (42), we need to compute $\langle K\rangle$ and $\left\langle K_{\mu \nu}\right\rangle u^{\mu}$. The trace of (42) and its contraction with $u^{\mu} u^{\nu}$ respectively give the system

$$
\begin{aligned}
& \frac{1}{\kappa_{[n]} \overline{\mathcal{T}}_{\infty}}[\mathcal{F}]=2(\hat{p}-1)\langle K\rangle+2(\hat{\rho}+\hat{p})\left\langle K_{\mu \nu}\right\rangle u^{\mu} u^{\nu}, \\
& \frac{n-2}{\kappa_{[n]} \overline{\mathcal{T}}_{\infty}}\left[\mathcal{F}_{\mu \nu}\right] u^{\mu} u^{\nu}=(1+\hat{\rho}-(\hat{\rho}+\hat{p})(n-2))\langle K\rangle+(1+\hat{\rho}-(1+2 \hat{\rho}+\hat{p})(n-2))\left\langle K_{\mu \nu}\right\rangle u^{\mu} u^{\nu} .
\end{aligned}
$$

This system of equations can be solved to give expressions for $\langle K\rangle$ and $\left\langle K_{\mu \nu}\right\rangle u^{\mu} u^{\nu}$ :

$$
\begin{aligned}
\langle K\rangle & =-\frac{(n-2)(\hat{\rho}+\hat{p})}{\kappa_{[n]} \overline{\mathcal{T}}_{\infty}(\hat{\rho}+1)^{2}(n-3)}\left[\mathcal{F}_{\mu \nu}\right] u^{\mu} u^{\nu}-\frac{(2 n-5) \hat{\rho}+(n-2) \hat{p}+n-3}{2 \kappa_{[n]} \overline{\mathcal{T}}_{\infty}(\hat{\rho}+1)^{2}(n-3)}[\mathcal{F}], \\
\left\langle K_{\mu \nu}\right\rangle u^{\mu} u^{\nu} & =-\frac{(n-2)(1-\hat{p})}{\kappa_{[n]} \overline{\mathcal{T}}_{\infty}(\hat{\rho}+1)^{2}(n-3)}\left[\mathcal{F}_{\mu \nu}\right] u^{\mu} u^{\nu}+\frac{(n-3) \hat{\rho}+(n-2) \hat{p}-1}{2 \kappa_{[n]} \overline{\mathcal{T}}_{\infty}(\hat{\rho}+1)^{2}(n-3)}[\mathcal{F}] .
\end{aligned}
$$

Substituting these two expressions back into the contraction of (42) with $u^{\nu}$ gives us an expression for $\left\langle K_{\mu \nu}\right\rangle u^{\nu}$

$$
-\left\langle K_{\mu \nu}\right\rangle u^{\nu}=\frac{n-2}{\kappa_{[n]} \overline{\mathcal{T}}_{\infty}(n-3)(\hat{\rho}+1)}\left[\mathcal{F}_{\mu \nu}\right] u^{\nu}+\frac{(n-2)(\hat{\rho}+\hat{p})}{\kappa_{[n]} \overline{\mathcal{T}}_{\infty}(n-3)(\hat{\rho}+1)^{2}}\left[\mathcal{F}_{\rho \sigma}\right] u^{\rho} u^{\sigma} u_{\mu}+\frac{(n-3) \hat{\rho}+(n-2) \hat{p}-1}{2 \kappa_{[n]} \overline{\mathcal{T}}_{\infty}(n-3)(\hat{\rho}+1)^{2}}[\mathcal{F}] u_{\mu}
$$

Substituting all of these back into (42) gives (43).

\section{APPENDIX B: WEYL CURVATURE FOR HYPER-SPHERICAL SPACETIMES}

The goal of this appendix is to give the element of derivation of equation (61) and to compute $\overline{\mathcal{W}}_{\mu \nu}$ as given in (60). For that purpose, we consider a $n$ dimensional Schwarzschild-de-Sitter bulk spacetime with line element

$$
\mathrm{d} s_{[n]}^{2}=-\mathcal{V}(r) \mathrm{d} t^{2}+\frac{\mathrm{d} r^{2}}{\mathcal{V}(r)}+r^{2}\left(\frac{\mathrm{d} \rho^{2}}{1-k \rho^{2}}+\rho^{2} \mathrm{~d} \ell_{[n-3]}^{2}\right)
$$

where $\mathrm{d} \ell_{[n]}^{2}$ is the measure on the unit $n$-sphere. The problem is that the line element for the unit $(n-3)$-sphere is not easy to write in a compact form. However, because of the symmetry of the spacetime, we need only to calculate 
the curvature at one point on the $(n-2)$-surfaces of spatial isometry. Thus, we perform a coordinate transformation to make these $(n-2)$-surfaces manifestly conformally flat and then use Cartesian coordinates, which makes it easy to generalize to higher dimensions. When $k=0$ this is trivial so we focus on the two cases $k= \pm 1$, where we define a new coordinate $\chi=\chi(\rho)$ such that the line element of these $(n-2)$-surfaces is

$$
\mathrm{d} s_{[n-2]}^{2}=r^{2}\left(\frac{\mathrm{d} \rho^{2}}{1-k \rho^{2}}+\rho^{2} \mathrm{~d} \ell_{[n-3]}^{2}\right)=\frac{r^{2}}{\left(\chi^{\prime}(\rho)\right)^{2}\left(1-k \rho^{2}\right)}\left(\mathrm{d} \chi^{2}+\rho^{2}\left(1-k \rho^{2}\right)\left(\chi^{\prime}(\rho)\right)^{2} \mathrm{~d} \ell_{[n-3]}^{2}\right),
$$

with prime denoting a derivative with respect to $\rho$. Thus, we need to choose $\chi(\rho)$ so that

$$
\rho^{2}\left(1-k \rho^{2}\right)\left(\chi^{\prime}(\rho)\right)^{2}=\chi(\rho)^{2} .
$$

For $k= \pm 1$, equation $(\mathrm{B} 3)$ can be integrated to give

$$
\chi(\rho)=k \frac{1-\sqrt{1-k \rho^{2}}}{\rho},
$$

It follows that the line element of the $(n-2)$-surfaces of spatial isotropy is

$$
\mathrm{d} s_{[n-2]}^{2}=\frac{2 r^{2}}{1+k \chi^{2}}\left(\mathrm{~d} \chi^{2}+\chi^{2} \mathrm{~d} \ell_{[n-3]}^{2}\right),
$$

which is manifestly conformally flat. Hence, writing (B5) as the appropriate conformal factor times the usual line element for $\mathbb{R}^{n-2}$, we conclude that the full $n$ dimensional line element can be written

$$
\mathrm{d} s_{[n]}^{2}=-\mathcal{V}(r) \mathrm{d} t^{2}+\frac{\mathrm{d} r^{2}}{\mathcal{V}(r)}+\frac{2 r^{2}}{\Omega^{2}}\left(\left(\mathrm{~d} x^{1}\right)^{2}+\cdots+\left(\mathrm{d} x^{n-2}\right)^{2}\right),
$$

where $2 \Omega^{2} \equiv 1+k\left(\left(x^{1}\right)^{2}+\cdots+\left(x^{n-2}\right)^{2}\right)$, which holds also for $k=0$.

We are now in a position to calculate the curvature tensors, which we do using the Cartan 2-form method (as outlined in ref. [23]). We write the metric (B6) in terms of a basis of orthonormal 1-forms as

$$
\mathrm{d} s^{2}=-\left(\boldsymbol{\omega}^{t}\right)^{2}+\left(\boldsymbol{\omega}^{r}\right)^{2}+\left(\boldsymbol{\omega}^{1}\right)^{2}+\cdots+\left(\boldsymbol{\omega}^{(n-2)}\right)^{2},
$$

where $\left\{\boldsymbol{\omega}^{t}, \boldsymbol{\omega}^{r}, \boldsymbol{\omega}^{i}\right\}$ is explicitly given by

$$
\boldsymbol{\omega}^{t} \equiv \mathcal{V}^{1 / 2} \mathrm{~d} t, \quad \boldsymbol{\omega}^{r} \equiv \frac{\mathrm{d} r}{\mathcal{V}^{1 / 2}}, \quad \boldsymbol{\omega}^{i} \equiv \frac{r}{\Omega} \mathrm{d} x^{i} .
$$

Note that boldface letters denote 1-forms and the indices do not denote components of these forms but labels. However, we will invoke the usual summation convention over repeated indices. The exterior derivatives of these 1-forms are

$$
\begin{aligned}
\mathrm{d} \boldsymbol{\omega}^{r} & =0 \\
\mathrm{~d} \boldsymbol{\omega}^{t} & =\frac{\mathcal{V}^{\prime}}{2 \mathcal{V}^{1 / 2}} \mathrm{~d} r \wedge \mathrm{d} t=\frac{\mathcal{V}^{\prime}}{2 \mathcal{V}^{1 / 2}} \boldsymbol{\omega}^{r} \wedge \boldsymbol{\omega}^{t}, \\
\mathrm{~d} \boldsymbol{\omega}^{i} & =\frac{1}{\Omega} \mathrm{d} r \wedge \mathrm{d} x^{i}-\frac{r}{\Omega^{2}} \frac{\mathrm{d} \Omega}{\mathrm{d} x^{j}} \mathrm{~d} x^{j} \wedge \mathrm{d} x^{i}=\frac{\mathcal{V}^{1 / 2}}{r} \boldsymbol{\omega}^{r} \wedge \boldsymbol{\omega}^{i}+\frac{1}{r} \frac{\mathrm{d} \Omega}{\mathrm{d} x^{j}} \boldsymbol{\omega}^{i} \wedge \boldsymbol{\omega}^{j} .
\end{aligned}
$$

Using the notation of ref. 23], we need to find the connection 1-forms $\boldsymbol{\omega}^{\mu}{ }_{\nu}$ which are antisymmetric (that is, $\boldsymbol{\omega}_{\mu \nu}=$ $-\boldsymbol{\omega}_{\nu \mu}$ ) and satisfy the first Cartan structure equation, $\mathrm{d} \boldsymbol{\omega}^{\mu}=-\boldsymbol{\omega}^{\mu}{ }_{\nu} \wedge \boldsymbol{\omega}^{\nu}$, for a torsion-free case, which can easily be seen to be satisfied by

$$
\begin{aligned}
\boldsymbol{\omega}^{t}{ }_{r} & =\boldsymbol{\omega}^{r}{ }_{t}=\frac{\mathcal{V}^{\prime}}{2 \mathcal{V}^{1 / 2}} \boldsymbol{\omega}^{t}=\frac{\mathcal{V}^{\prime}}{2} \mathrm{~d} t, \\
\boldsymbol{\omega}^{t}{ }_{i} & =\boldsymbol{\omega}^{i}{ }_{t}=0 \\
\boldsymbol{\omega}^{i}{ }_{r} & =-\boldsymbol{\omega}^{r}{ }_{i}=\frac{\mathcal{V}^{1 / 2}}{r} \boldsymbol{\omega}^{i}=\frac{\mathcal{V}^{1 / 2}}{\Omega} \mathrm{d} x^{i}, \\
\boldsymbol{\omega}^{i}{ }_{j} & =\frac{1}{r}\left(\frac{\mathrm{d} \Omega}{\mathrm{d} x^{i}} \boldsymbol{\omega}^{j}-\frac{\mathrm{d} \Omega}{\mathrm{d} x^{j}} \boldsymbol{\omega}^{i}\right)=\frac{1}{\Omega}\left(\frac{\mathrm{d} \Omega}{\mathrm{d} x^{i}} \mathrm{~d} x^{j}-\frac{\mathrm{d} \Omega}{\mathrm{d} x^{j}} \mathrm{~d} x^{i}\right) .
\end{aligned}
$$


The Cartan curvature two-forms are then given by the second Cartan structure equation

$$
\Re^{\mu}{ }_{\nu} \equiv \mathrm{d} \boldsymbol{\omega}^{\mu}{ }_{\nu}+\omega^{\mu}{ }_{\rho} \wedge \boldsymbol{\omega}^{\rho}{ }_{\nu},
$$

from which the components of the Riemann tensor can be read off via

$$
\Re^{\mu}{ }_{\nu}=\mathcal{R}^{\mu}{ }_{\nu \rho \sigma} \boldsymbol{\omega}^{\rho} \wedge \boldsymbol{\omega}^{\sigma} .
$$

Because of the symmetry of the Schwarzschild-de-Sitter spacetime, it is only necessary to evaluate the curvature 2 -forms at a point, $x^{1}=\cdots=x^{(n-2)}=0$ say, giving

$$
\Re_{r}^{t}=-\frac{\mathcal{V}^{\prime \prime}}{2} \boldsymbol{\omega}^{t} \wedge \boldsymbol{\omega}^{r}, \quad \Re_{i}^{t}=-\frac{\mathcal{V}^{\prime}}{2 r} \boldsymbol{\omega}^{t} \wedge \boldsymbol{\omega}^{i}, \quad \Re^{r}{ }_{i}=-\frac{\mathcal{V}^{\prime}}{2 r} \boldsymbol{\omega}^{r} \wedge \boldsymbol{\omega}^{i}, \quad \Re^{i}{ }_{j}=\left(\frac{k}{r^{2}}-\frac{\mathcal{V}}{r}\right) \boldsymbol{\omega}^{i} \wedge \boldsymbol{\omega}^{j} .
$$

Hence the non-zero components of the Riemann tensor are

$$
\mathcal{R}^{t r}{ }_{t r}=-\frac{\mathcal{V}^{\prime \prime}}{2}, \quad \mathcal{R}^{t i}{ }_{t j}=\mathcal{R}^{r i}{ }_{r j}=-\frac{\mathcal{V}^{\prime}}{2 r} \delta^{i}{ }_{j}, \quad \mathcal{R}^{i j}{ }_{k l}=2 \frac{k-\mathcal{V}}{r^{2}} \delta^{[i}{ }_{[k}{ }^{j]}{ }_{l]},
$$

giving a the non-zero components of the Ricci tensor

$$
\mathcal{R}^{t}{ }_{t}=\mathcal{R}_{r}^{r}=-\frac{\mathcal{V}^{\prime \prime}}{2}-(n-2) \frac{\mathcal{V}^{\prime}}{2 r}, \quad \mathcal{R}^{i}{ }_{j}=\left((n-3) \frac{k-\mathcal{V}}{r^{2}}-\frac{\mathcal{V}^{\prime}}{r}\right) \delta^{i}{ }_{j},
$$

and the Ricci scalar

$$
\mathcal{R}=-\mathcal{V}^{\prime \prime}-2(n-2) \frac{\mathcal{V}^{\prime}}{r}+(n-2)(n-3) \frac{k-\mathcal{V}}{r^{2}} .
$$

The Weyl tensor can then be calculated by the standard formula

$$
\mathcal{C}^{\alpha \beta}{ }_{\gamma \delta}=\mathcal{R}^{\alpha \beta}{ }_{\gamma \delta}-\frac{4}{n-2} \delta^{[\alpha}{ }_{[\gamma} \mathcal{R}^{\beta]}{ }_{\delta]}+\frac{2}{(n-1)(n-2)} \delta_{[\gamma}^{[\alpha} \delta^{\beta]}{ }_{\delta]} \mathcal{R},
$$

so that its non-zero components are

$$
\mathcal{C}^{t r}{ }_{t r}=(n-2) \mathcal{W}, \quad \mathcal{C}^{t i}{ }_{t j}=\mathcal{C}^{r i}{ }_{r j}=-\mathcal{W} \delta^{i}{ }_{j}, \quad \mathcal{C}^{i j}{ }_{k l}=\frac{4 \mathcal{W}}{n-3} \delta^{[i}{ }_{[k} \delta^{j]}{ }_{l]}
$$

where the Weyl scalar $\mathcal{W}$ is obtained to be

$$
\mathcal{W}=\frac{n-3}{(n-1)(n-2)}\left(-\frac{\mathcal{V}^{\prime \prime}}{2}+\frac{\mathcal{V}^{\prime}}{r}+\frac{k-\mathcal{V}}{r^{2}}\right)=(n-3) \frac{G_{[n]} \mathcal{M}}{r^{n-1}}
$$

hence reducing to the expression (61) on the brane.

We can also determine the components of the normal vector $n^{\mu}$ in this coordinate system by contracting it with the timelike killing covector of the Schwarzschild-de-Sitter spacetime $k=d t$ and noting that $k_{\mu} n^{\mu}$ must be constant along geodesics and so depends only on $\tau$ in the brane-based coordinates [17]. With the normalization chosen in (55), we have $k_{\mu} n^{\mu}=\mathrm{d} a / \mathrm{d} \tau$, which determines the $t$-component of $n^{\mu}$ in the static coordinates we have used to compute the Weyl tensor. As $n^{\mu}$ is a unit vector and is orthogonal to the $(n-2)$-surfaces of spatial isometry, its components are

$$
n^{t}=\frac{\mathrm{d} a}{\mathrm{~d} \tau}, \quad n^{r}=\mathcal{V}^{1 / 2}\left(1+\mathcal{V}\left(\frac{\mathrm{d} a}{\mathrm{~d} \tau}\right)^{2}\right)^{1 / 2}, \quad n^{i}=0 .
$$

This allows us to calculate $\overline{\mathcal{W}}_{\mu \nu}$ which, in the brane-based coordinates (55), has the form given in (60).

[1] N. Arkani-Hamed, S. Dimopolous, and G. Dvali, Phys. Lett. B429, 263 (1998). 
[2] L. Randall and R. Sundrum, Phys. Rev. Lett. 83, 3370 (1999).

[3] L. Randall and R. Sundrum, Phys. Rev. Lett. 83, 4690 (1999).

[4] P. Binétruy, C. Deffayet, and D. Langlois, Nucl. Phys. B565, 269 (2000).

[5] J.M. Cline, C. Grojean, and G. Servant, Phys. Rev. Lett. 83, 4245 (1999).

C. Csaki, M. Graesser, C. Kolda, and J. Terning, Phys. Lett. B462, 34 (1999).

P. Binétruy, C. Deffayet, U. Ellwanger, and D. Langlois, Phys. Lett. B477, 285 (1999).

E.E. Flannagan, S.-H. Tye, and I. Wasserman, Phys. Rev. 62, 044039 (2000).

[6] N. Deruelle and J. Katz, gr-qc/0104007.

[7] R. Kallosh and A. Linde, JHEP 0002, 005 (2000).

K. Behrndt and M. Cvetič, Phys. Rev. D61, 101901 (2000).

G.W. Gibbons and N.D. Lambert, Phys. Lett. B488, 90 (2000).

J. Maldacena and C. Nuñes, Int. J. Mod. Phys. A16, 822-855 (2001).

[8] R. Altendorfer, J. Bagger and D. Nemeschansky, hep-th/0003117.

A. Falkowski, Z. Lalak and S. Pokorski, Phys. Lett. B491, 172-182 (2000).

[9] T. Shiromizu, K. Maeda, and M. Sasaki, Phys. Rev. D62, 024012 (2000).

[10] R. Maartens, Phys. Rev. D62, 084023 (2001).

[11] P. Hořava and E. Witten, Nucl. Phys. B460, 500 (1996).

P. Hořava and E. Witten, Nucl. Phys. B475, 94 (1996).

[12] J. Polchinski, Phys. Rev. Lett. 75, 4724-4727 (1995).

[13] B. Carter, gr-qc/0012036. (to appear in Int. J. Mod. Phys.)

[14] R.A. Battye and B. Carter, hep-th/0101061]. (to appear in Phys. Lett. B)

[15] T. Gherghetta and M. Shaposhnikov, Phys. Rev. Lett. 85, 240 (2000).

T. Gherghetta, E. Roessl, and M. Shaposhnikov, Phys. Lett. B491, 353 (2000).

M. Gogberashvili and P. Midodashvili, hep-ph/0005298.

[16] P. Krauss, JHEP 9912, 011 (1999).

D. Ida, JHEP 0009, 014 (2000).

N. Deruelle and T. Dolezel, Phys. Rev. D62, 10350 (2000).

H. Stoica, S.-H. Tye, and I. Wasserman, Phys. Lett. B482, 205 (2000).

A.-C. Davis, S. Davis, W.B. Perkins, and I.R. Vernon, Phys. Rev. D63, 083518 (2001).

W. Perkins, Phys. Lett. B504, 28-32 (2001).

[17] B. Carter and J.-P. Uzan, gr-qc/010101d. (to appear in Nucl. Phys. B)

[18] A. Kehagias and E. Kiritsis, JHEP 9911, 022 (1999).

G. Kofinas, hep-th/0103045.

[19] D. Wands and K. Maeda, Phys. Rev. D62, 124009 (2000).

[20] A. Mennim and R.A. Battye, hep-th/0008192. (to appear in Class. Quant. Grav.)

[21] J. Garriga and T. Tanaka, Phys. Rev. Lett. 84, 2778 (2000).

C. Csaki, J. Erlich, T. Hollowood, and Y. Shirman, Nucl. Phys. B581, 309 (2000).

S.B. Gidding, E. Katz, and L. Randall, JHEP 0003, 023 (2000).

C. Csaki, J. Erlich, and T. Hollowood, Phys. Rev. Lett. 84, 5932 (2000).

[22] J.A. Schouten, Ricci Calculus (Springer, 1954).

[23] C.W. Misner, K.S. Thorne, and J.A. Wheeler, Gravitation (W.H. Freeman and Company, 1973).

[24] W. Israel [1966], Nuovo Cimento 44B, 1. Erratum: Nuovo Cimento 48B, 463.

[25] G. Darmois, Mémorial des sciences mathématiques XXV (1927)

[26] K. Lanczos (1922) unpublished — published, Ann. Phys. (Leipzig) 74, 518 (1924).

[27] N. Sen, Ann. Phys. (Leipzig) 73, 365 (1924).

[28] R.A. Battye, B. Carter, A. Mennim, and J.-P. Uzan, in preparation.

[29] P. Bowcock, C. Charmousis, and R. Gregory, Class. Quant. Grav. 17, 4745 (2000). 